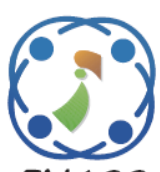

\title{
New Interest Points Detector for 3D Objects Recognition
}

\author{
Youness Abouqora $^{1 *} \quad$ Omar Herouane $^{1} \quad$ Lahcen Moumoun $^{1} \quad$ Taoufiq Gadi $^{1}$ \\ ${ }^{1}$ Laboratory of Informatics, Imaging, and Modeling of Complex Systems (LIIMSC), \\ Faculty of Sciences and Techniques, Hassan 1st University, Settat, Morocco \\ * Corresponding author's Email: yabouqora@gmail.com
}

\begin{abstract}
The interest point detection is a technique for reducing the amount of data that needs to be processed in certain applications, this technique is widely used in 2D and 3D image analysis. In this paper, we introduce a new method for detecting the mesh interest points based on geometric measures. The key point of this new approach is to extract a satisfactory 3D interest points with a high repeatability and relevant distribution of the whole object. It consists of two steps: an interest point detection and a refinement post-processing. First, we compute the Harris operator function, that is robust to several transformations, around each vertex to selects the maximal responses as candidates of 3D interest points. Second, we use the shape index-based optimization method to select the final interest points with high curvedness. The performance of the proposed method is evaluated and it proves encouraging results.
\end{abstract}

Keywords: 3D objects, Computer vision, 3D interest points detection, Harris operator, Shape index, Repeatability rate, IOU metric.

\section{Introduction}

With the rapid development of 3D image representation and acquisition techniques, many 3D image's data bases have been created and widely applied in our daily life. 3D interest point is one of the most significant local feature in these images, it can be effectively used to simplify the data in order to remove the redundancy. It lays on the foundation of relative computer vision and graphics applications, such as registration [1], shape retrieval [2,3], object recognition [4], mesh segmentation [5] and simplification [6] etc. Compared with 2D interest point detection algorithms, like SIFT [7], Harris [8], SODC [9] and FAST [10], the 3D one has many challenging tasks due to the presence of noise, occlusion, clutter and a wide range of shape transformations.

Several approaches have been proposed to extend the developed interest point detectors in the 2D images to the 3D field. Most of them have many drawbacks, such as the detection of few key points, particularly on the less curved parts of the 3D object. Moreover, they could not exploit the scale information encoded in the local geometric structures to detect the inherent scale of a key point and they are also sensitivity to noise.

Motivated by these limitations, we propose a highly discriminative and robust detector. First, we use a Harris operator that determines the neighborhood for each vertex to fit a quadratic patch. Then, computing a derivatives functions to select the local maxima of responses as candidate sets using clustering techniques. Second, we compute the curvature values around the result set points, using shape index, to select the interest points with the more significant curvature. Referring to the benchmark and the evaluation study [31], our approach results prove that the detected key points are quite uniformly distributed over the surface, repeatable and computationally efficient.

Furthermore, our proposed detector can be used later to develop a 3D objects' descriptors. Those descriptors will be used to feed a machine learning algorithm as feature vectors to recognize the whole $3 \mathrm{D}$ objects or object-parts generated by automatic segmentation's algorithms and compare the results 
with some new relevant algorithms like that of Herouane et al. [13].

The remainder of this paper is organised as follows. In Sec. 2 we discuss some relevant research works in the literature. The 3D interest points feature computation is described in Sec. 3. Our experimental setting and results are elaborated in Sec. 4. We conclude the paper in Sec. 5.

\section{3D Interest points detection}

There is no exact definition for interest points up to now and different applications may have different requirements for these points. For example, some applications are inclined to choose the interest points with high repeatability or prefer them with a higher discriminability and also others may require them with semantic information, such as Schelling point [11]. Thus, when designing the interest point detection algorithms, we usually need to define the proper interest point response function according to the practical situation.

Fortunately, researchers have proposed many 3D interest point detection algorithms to overcome the above-mentioned difficulties, most of them are based on geometric criteria's [6, 12, 21]. Godila in [20] converted the 3D mesh model into voxel grid representation and proposed a method for detecting 3D salient local features inspired by the SIFT algorithm [7], in which 3D DoG (Difference of Gaussian) operator is employed to detect robust 3D interest points. The authors of $[15,16,17,18]$ utilized similar ways to detect 3D interest point. Sipiran and Bustos proposed an effective and efficient extension of the Harris operator [8] for 3D objects [14]. Lee in [6] introduced mesh saliency as a measure of regional importance for 3D meshes, where they define mesh saliency in a scale-dependent manner using a centersurround operator on Gaussian-weighted mean curvatures. Holte addressed the problem of detecting 3D interest points using Difference-of-Normal operator [12]. Castellani proposed a salient point detection algorithm where sparse 3D key points were selected robustly by exploiting the visual saliency principles on 3D mesh [20]. There exist also some methods for detecting 3D interest point in spectrum domain instead of spatial domain [2, 22, 22]. Besides, some researchers formulated 3D interest point detection as a supervised binary classification problem and used learning methods to detect these points $[25,26]$.

\section{Proposed approach}

The proposed detector uses both the Harris matrix and the measure of curvature. First, we select the candidate interest points using 3D Harris detector that works in a vertex-wise manner in order to compute the response function for each vertex. It aims at considering a local neighborhood around a vertex as an image, so it is possible to apply the well-known Harris operator. Second, in order to pick out the most repeatable and salient set of 3D points, we propose to compute the shape index. To refine them, we select the surfaces with the extremum curvature.

\subsection{D Harris operator}

The 3D Harris detector is the 3D extension of the 2D corner detection method of Harris and Stephens [8]; it is based on the first order derivatives in two orthogonal directions on the 3D surface. For each vertex $v$ associated to its Harris operator, the value is calculated as following Eq. (1):

$$
h(v)=\operatorname{det}(E)-k(\operatorname{tr}(E))^{2}
$$

where $E$ represents the auto-correlation matrix of derivatives function and $k$ a parameter that needs to be experimentally tuned.

The authors of [14], propose two ways to select the interest points of a given object. First, the vertices which are local maximum are extracted. To do so, they select a vertex $v$ which holds the following condition Eq. (2):

$$
h(v)>h(w), \forall w \in \operatorname{ring} 1(v) .
$$

Second, two approaches to select the final set of interest points are proposed:

- Select the points with the highest Harris response. We can pick a constant fraction of interest points depending on the application. In this proposal, we obtain the points with higher saliency and therefore, some portions of the object do not have interest points.

- Representatives of Interest Points Clusters. This approach can be used when we want to get a good distribution of interest points in the object surface. This proposal consists of two steps. First, we sort the pre-selected interest points according to their Harris operator value in decreasing order. Second, we apply Algorithm 1 to cluster the sorted points and select the final set of interest points. 


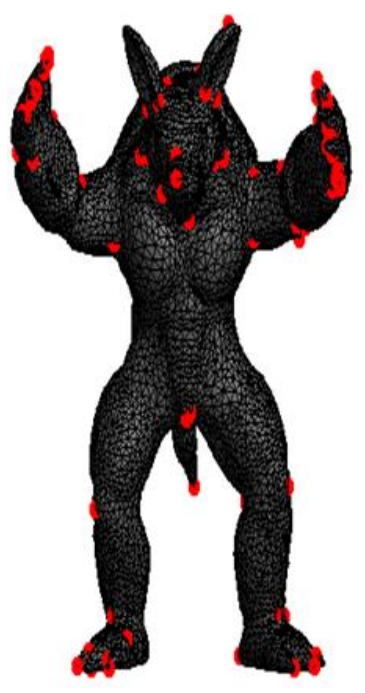

(a)

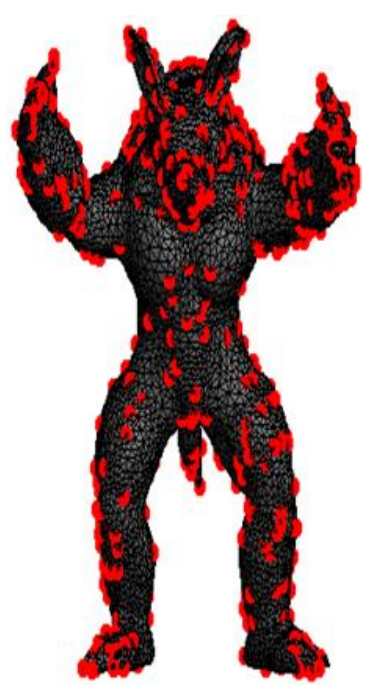

(b)
Figure.1 "Armadillo" model: (a) selected points with highest Harris response and (b) selected points by clustering

\section{Algorithm 1: Interest Points clustering}

Input: Set $\mathrm{P}$ of pre-selected interest points in decreasing order of Harris Operator value Output: Candidates set of interest points

- Let $\boldsymbol{Q}$ be a set of points

- $Q \leftarrow \varnothing$

- for $\boldsymbol{i} \leftarrow \mathbf{1}$ to $|P|$ do

- if $\boldsymbol{m i n}_{j \in[1,|Q|]}\left\|P_{i}-Q_{j}\right\|_{2}>\rho$ then

- $\boldsymbol{Q} \leftarrow \boldsymbol{Q} \cup\left\{\boldsymbol{P}_{i}\right\}$

- End if

- end for

- return $\boldsymbol{Q}$

The value of $\rho$ can be considered as a fraction of the diagonal of the object bounding rectangle and it has effect in the number of returned interest points.

Fig.1 shows the result of the two options used to select interest points. According to these results, we have choosing to use the clustering approach because its good representativity for the entire object.

\subsection{Refinement process}

The Harris clustering approach is interesting for applications requiring points well distributed over the whole surface. Nevertheless, as the process is based on grouping vertices with high responses, this step is not necessarily robust according to the repeatability criterion. Thus, we propose a computation of the shape index to classify the 3D shape surface and select the final interests point with the high curvature. The shape index (SI) as proposed by Koenderink [23] is a quantitative measure of the surface shape at a point $\mathrm{p}$, and is defined by Eq. (3):

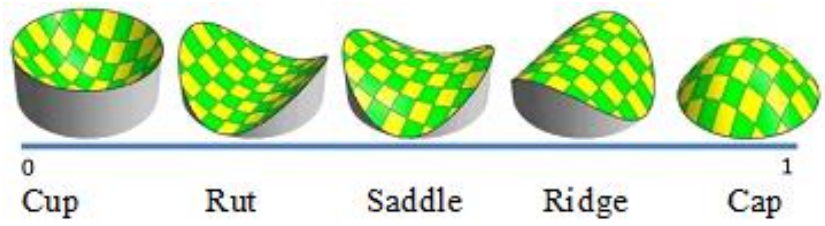

Figure.2 Illustration of shape index scale divided into five categories

$$
S I(p)=\frac{1}{2}-\frac{1}{\pi} \arctan \left(\frac{K(p)_{1}+K(p)_{2}}{K(p)_{1}-K(p)_{2}}\right)
$$

Where $k(p)_{1}$ and $k(p)_{2}, k(p)_{1}>k(p)_{2}$ maximum and minimum principal curvatures at a point $\mathrm{p}$, respectively. With this definition, all shapes are mapped into the interval $[0,1]$ where every distinct surface shape corresponds to a unique value of SI (except for planar surfaces, which will be mapped to the value 0.5 , together with saddle shapes). Larger shape index values represent convex surfaces and smaller shape index values represent concave surfaces Fig. 2. The main advantage of this measure is the invariance to orientation and scale.

To select the most relevant candidates of 3D interest points of a mesh, we use the shape index to specify them with a higher curvature. To perform this, we proceed to detect the interest points with the same salient shape features of the starting points that should be eliminated in its neighborhoods.

Algorithm 2: Our Interest Points detection using shape index (SI)

Input: Q interest points' Candidates extracted by Harris clustering and $\mathrm{V}$ the mesh vertices

Output: Final interest points set

- Let $\boldsymbol{F}$ be a set of points

- $\boldsymbol{F} \leftarrow \varnothing$

- $S I \leftarrow\{S I(\mathrm{~V})\}$

- for $\boldsymbol{i} \leftarrow \mathbf{1}$ to $|Q|$ do

- if $\| S I\left(Q_{i}\right)-S I\left(\operatorname{vring} 3\left(Q_{i}\right) \|>\right.$ threshold then

- $\boldsymbol{F} \leftarrow \boldsymbol{F} \cup\left\{\boldsymbol{Q}_{i}\right\}$

- End if

- end for

- $\operatorname{return} \boldsymbol{F}$

Based on [23] categorization of surfaces, the threshold is fixed at 0.3 to determine the curvatures variation between concavity and convexity based on shape index values. 

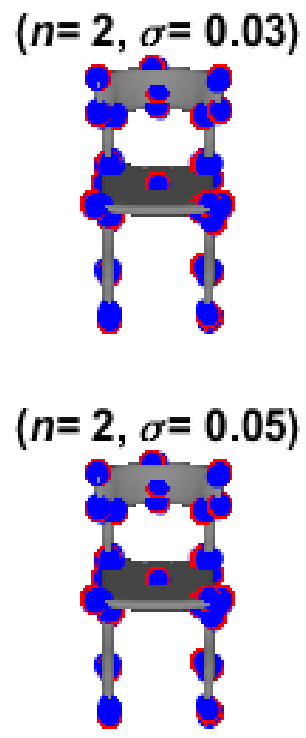
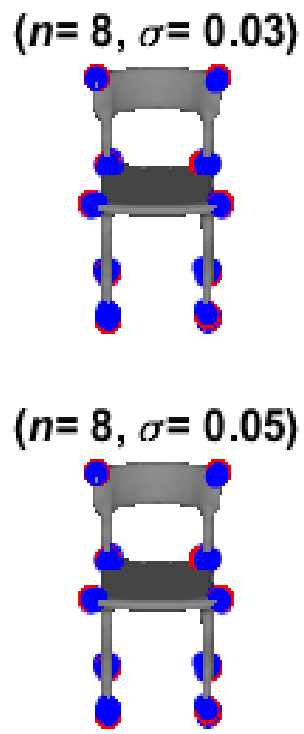
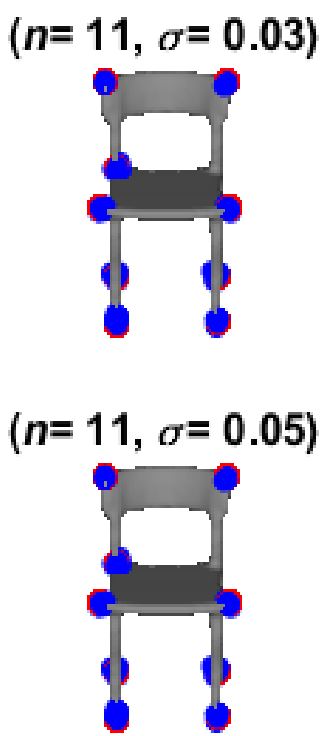

Figure.3 3D interest points of ground truth (red dots) of "chair" mesh m model marked by different human subjects n (blue dots) and radius $\sigma$

\section{Experimental results}

To access the effectiveness of the interest point detection algorithms, different authors used a variation of techniques to perform this task. The authors of $[28,30]$ measure the repeatability rate according to varying factors, which are usually designed for some special tasks like shape retrieval [30]. Another research work [31] utilizes the human generated ground truth data to evaluate the performance of 3D interest point detection algorithms. In this paper, we only focus on the problem of 3D interest point detection. Therefore, we evaluate the performance of our proposed detector and compare it with five 3D interest point detection algorithms, named respectively, 3D Harris [14], HKS [22], Salient Points [18], Mesh Saliency [6] and Scale Dependent Corners [19].

\subsection{Dataset}

The used dataset consists of two sub-datasets (dataset A and B) [31]. The first one (dataset A) is constituted by 24 triangular mesh models and annotated by 23 human subjects. The other one (dataset B) is constituted by 43 triangular mesh models and annotated by 16 human subjects. Groundtruth points are constructed from the human-marked points based on two criteria: radius of an interest region $s$ and the number of users $n$ who marked a point within the radius. In addition to the location, the prominence of a ground-truth point is included as the number of humans who have marked it within its local neighborhood. An example of interest points detected on a model from dataset B are shown in Fig. 3.

\subsection{Evaluations metrics}

On this dataset, evaluation was performed to test the compatibility of each algorithm with human perception. This experiment is designed to test an interest point detector in terms of distinctiveness, which demonstrates the ability of an interest point to detect representative and characteristic points on a surface.

Our evaluation was performed on each single instance of the model using human generated groundtruth. Three measures are used [31] i.e., false positive errors FPE, false negative errors FNE and weighted miss error WME. But, FPE and FNE can be misleading in isolation as discussed in [29]. So, Teran [26] adopted the Intersection Over Union (IOU) [32] which combines False Positive FP, False Negative FN and True Positive TP together as their main metric to evaluate the performance of the 3D interest point algorithms. In this paper, we also adopt the IOU metric as the main evaluation metric to evaluate the performance of our proposed interest points algorithm and other six state-of-the-art methods.

Let $G M(n ; s)$ be the set of ground-truth points on a model $M$ and $M_{D}$ be the set of interest points detected by an algorithm. A point $\mathrm{v}$ is considered to 


\section{Ground truth}

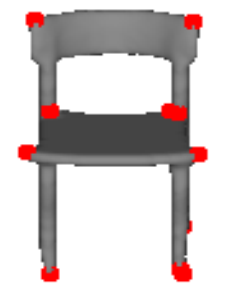

\section{D SI-Harris}

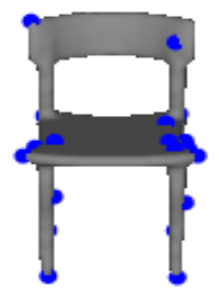

3D Harris

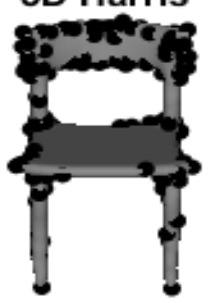

Mesh saliency

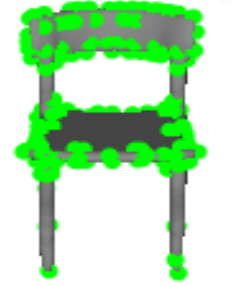

SD corners

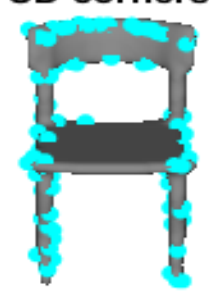

\section{Salient points}

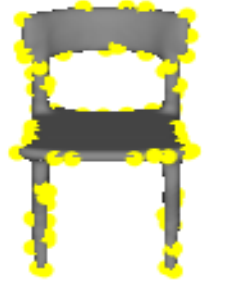

HKS

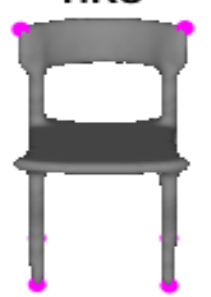

Figure.4 A 3D interest points of chair model in dataset A detected by the six algorithms and ground truth $n=8, \sigma=$ 0.03

be 'correctly detected' if there is a detected point within the geodesic ball $N r(v)$, where the parameter $r$ is the localization error tolerance. Given the number of ground-truth points $N_{G T}$, the points detected by an algorithm $N_{D}$ and the correctly detected points $N_{C}$, IOU score to determine error tolerance $r$, it can be calculated by Eq. (4):

$$
I O U(r)=\frac{T P}{F N+F P+T P}
$$

where $F P=N_{D}-N_{C}$ is the number of false positives and $F N=N_{G T}-N_{C}$ represents the number of false negatives. TP $=N_{C}$ is the true positives. $N_{G T}$ is the number of ground truth points, $N_{C}$ is the number of correctly detected points and $N_{D}$ denotes the number of detected interest points by the algorithm.

\subsection{Performance evaluations}

To access the effectiveness of the interest point detection algorithms, we display the performance results of six algorithms on Dataset A and Dataset B in terms of IOU metric. It is important to note that the 3D interest points detected by these 3D interest point detection algorithms are constant when parameters $n / \sigma$ varies, but the ground truth is variable when parameters $n / \sigma$ varies according to [31].

Fig. 4 shows the obtain results, where 3D interest points of the "chair" model detected by the six algorithms above are displayed. The HKS [22] algorithm can detect a small number of significant salient points but can't do even slightly for more ambiguous interest points. While, for a recognition goal that detects the salient points with a high robustness and repeatability, we except the HKS from the evaluation process. 


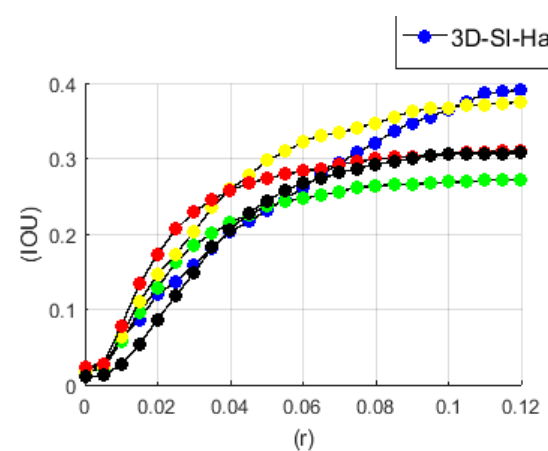

$(n=2, \sigma=0.03)$

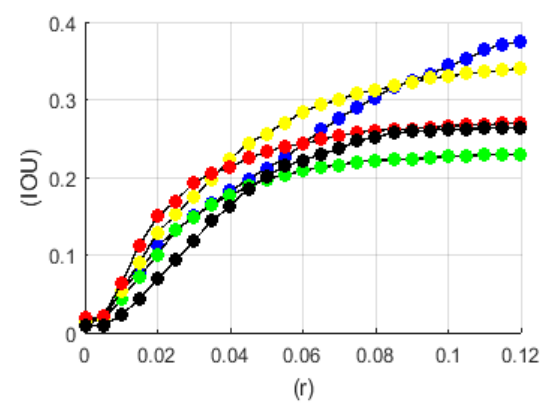

$(n=2, \sigma=0.05)$

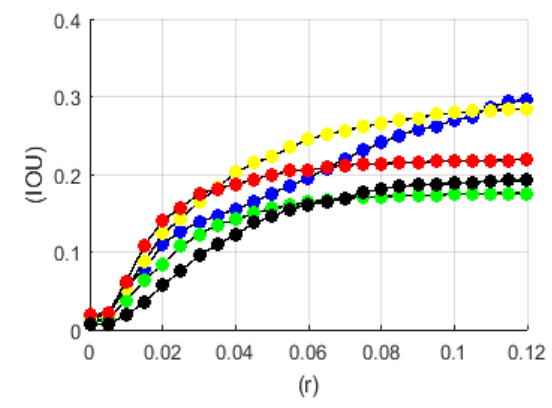

$(n=8, \sigma=0.05)$

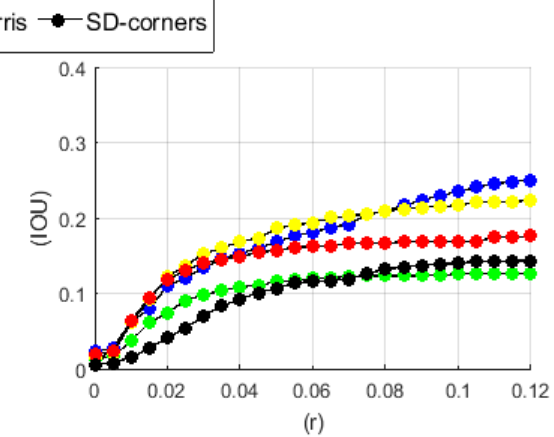

$(n=11, \sigma=0.03)$

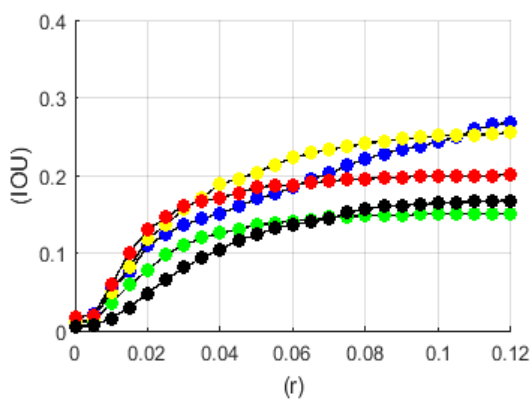

$(n=11, \sigma=0.05)$

Figure.5 IOU scores for Dataset A at various parameter pairs $n / \sigma$ for different localization error tolerance $\mathrm{r}$.

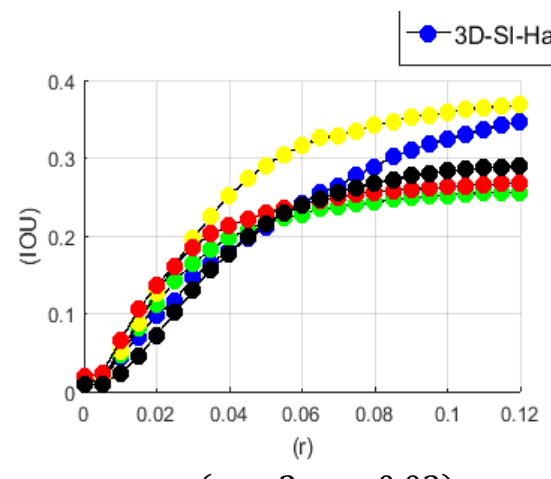

$$
(n=2, \sigma=0.03)
$$

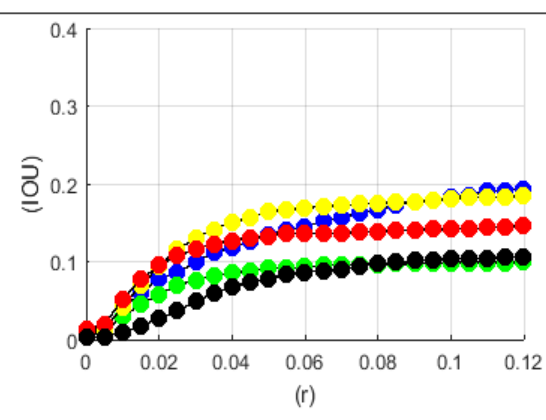

$(n=8, \sigma=0.03)$

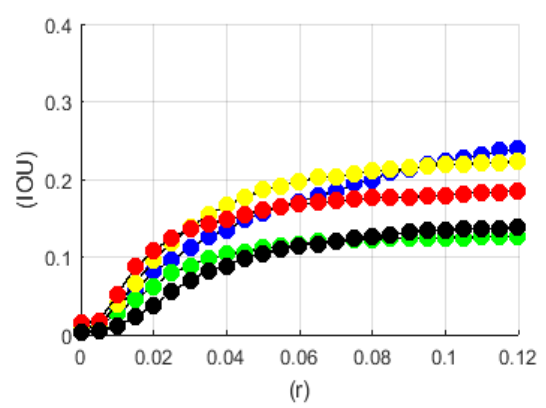

$(n=8, \sigma=0.05)$

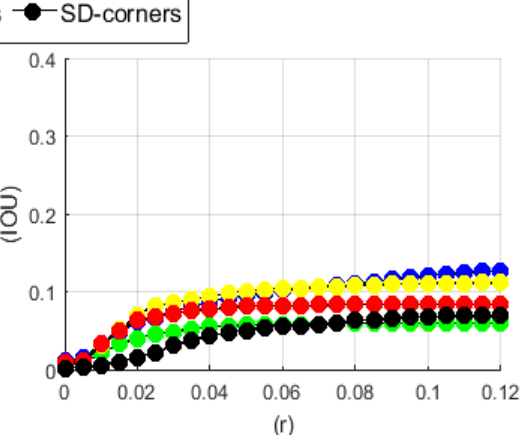

$(n=11, \sigma=0.03)$

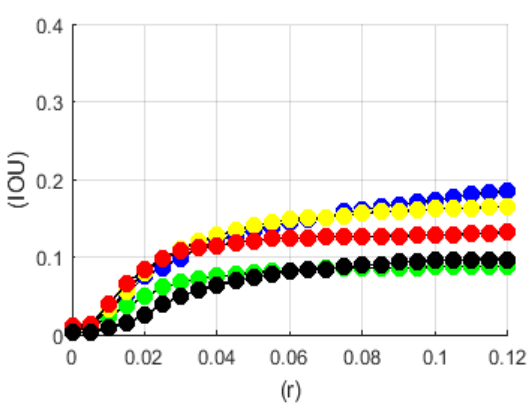

$(n=11, \sigma=0.05)$

Figure.6 IOU scores for Dataset B at various parameter pairs $n / \sigma$ for different localization error tolerance $\mathrm{r}$

In order to quantitatively analyze the performance of the five algorithms, we first test the IOU scores for dataset $\mathrm{A}$ and dataset $\mathrm{B}$ at various parameter $\frac{n}{\sigma}$ pairs. Fig. 5 and Fig. 6 show the IOU scores at several parameters $\frac{n}{\sigma}$ pairs for dataset A and 
Table 1. Table Average IOU on Dataset A $n \in$

$\{2,3, \ldots, 23\}, \sigma \in\{0.01,0.02, \ldots, 0.1\}$ and Dataset B $n \in\{2,3, \ldots, 16\}, \sigma \in\{0.01,0.02, \ldots, 0.1\}$

\begin{tabular}{|c|c|c|}
\cline { 2 - 3 } \multicolumn{1}{c|}{} & Dataset A & Dataset B \\
\hline Mesh saliency & 0.0722 & 0.0726 \\
\hline SD corners & 0.0815 & 0.0755 \\
\hline Salient points & 0.1334 & 0.1138 \\
\hline 3D SI-Harris & $\mathbf{0 . 1 5 0 8}$ & $\mathbf{0 . 1 5 8 5}$ \\
\hline
\end{tabular}

dataset $\mathrm{B}$ respectively, in which we set $n \in$ $\{2,8,11\}, \sigma \in\{0.03,0.05\} \quad$ and $\quad r \in$ $\{0.005,0.01,0.015, \ldots, 0.12\}$.

From Fig.5, we can see that our approach (3D SIHarris) detector performs well in the majority of the cases mostly for the one where $\mathrm{n}$ is high. When localization error tolerance $r$ is relatively large, the superiority of the 3D SI-Harris is more significant.

To reach an overall ranking, we calculate the average IOU score over all $n / \sigma$ settings in Dataset A. Table 1. displays the average IOU scores of Dataset $A$ and Dataset B. We can remark that the 3D SIHarris based 3D interest point detector performs well in term of IOU evaluation metric.

\section{Conclusion}

In this paper, we present a new method for $3 \mathrm{D}$ interest point's detection applied on 3D shapes. Our approach captures simultaneously the local geometric measures and global structural information of a surface. It extracts interest points with a good distribution over the surface with less sensitivity to noise and more robustness to several transformations.

The experimental results demonstrate obviously the effectiveness of our approach in terms of IOU metric compared to previous proposed algorithms.

In our future works, we attempt to combine our interest point's detector with a local surface descriptor and test it on some applications such as surface registration, shape tracking and shape retrieval of the $3 \mathrm{D}$ objects.

\section{References}

[1] N. Gelfand, N.J. Mitra, L.J. Guibas, and H. Pottmann, "Robust global registration", In: Symposium on geometry processing, pp.5-5, 2005.

[2] J. Hu and J. Hua, "Salient spectral geometric features for shape matching and retrieval", The
Visual Computer, Vol.25, No.5-7, pp.667-675, 2009.

[3] T. Funkhouser and M. Kazhdan, "Shape-based retrieval and analysis of 3D models", In: $A C M$ SIGGRAPH 2004 Course Notes, pp.16-16, 2004.

[4] A.E. Johnson and M. Hebert, "Using spin images for efficient object recognition in cluttered 3D scenes", IEEE Transactions on Pattern Analysis and Machine Intelligence, Vol.21, No.5, pp.433-449, 1999.

[5] S. Katz, G. Leifman, and A. Tal, "Mesh segmentation using feature point and core extraction", The Visual Computer, Vol.21, No.810, pp.649-658, 2005.

[6] C.H. Lee, A. Varshney, and D.W. Jacobs, "Mesh saliency", ACM Transactions on Graphics, Vol.24, No.3, pp.659-666, 2005.

[7] D.G. Lowe, "Distinctive image features from scale-invariant keypoints," International Journal of Computer Vision, Vol.60, No.2, pp.91-110, 2004.

[8] C.G. Harris and M. Stephens, "A combined corner and edge detector", In: Alvey Vision Conference, pp.10-5244, 1988.

[9] X. Lin, C. Zhu, Q. Zhang, X. Huang, and Y. Liu, "Efficient and robust corner detectors based on second order difference of contour", IEEE Signal Processing Letters, Vol.24, N.9, pp.1393-1397, 2017.

[10] E. Rosten and T. Drummond, "Machine learning for high-speed corner detection", In: Proc. of European Conference on Computer Vision, pp.430-443, 2006.

[11] X. Chen, A. Saparov, B. Pang, and T. Funkhouser, "Schelling points on 3D surface meshes", ACM Transactions on Graphics, Vol.31, No.4, pp.29-29, 2012.

[12] M.B. Holte, "3D interest point detection using local surface characteristics with application in action recognition", In: IEEE international Conference on Image Processing, Paris, France, pp. 5736-5740, 2014.

[13] O. Herouane, L. Moumoun, T. Gadi, and M. Chahhou, "A Hybrid Boosted-SVM Classifier for Recognizing Parts of 3D Objects", International Journal of Intelligent Engineering and Systems, Vol.11, No.2, pp.102-110, 2018.

[14] I. Sipiran and B. Bustos, "Harris 3D: a robust extension of the harris operator for interest point detection on 3D meshes", The Visual Computer, Vol.27, No.11, pp.963-963, 2011.

[15] A. Zaharescu, E. Boyer, and R. Horaud, "Keypoints and local descriptors of scalar functions on 2D manifolds", International 
Journal of Computer Vision, Vol.100, No.1, pp.78-98, 2012.

[16] T. Darom and Y. Keller, "Scale-invariant features for 3D mesh models", IEEE Transactions on Image Processing, Vol.21, No.5, pp.2758-2769, 2012.

[17] B. Rister, D. Reiter, H. Zhang, D. Volz, M. Horowitz, R. E. Gabr, and J. R. Cavallaro, "Scale- and orientation-invariant keypoints in higher-dimensional data", In: Proc. of IEEE International Conference on Image Processing, pp.3490-3494, 2015.

[18] U. Castellani, M. Cristani, S. Fantoni, and V. Murino, "Sparse points matching by combining 3D mesh saliency with statistical descriptors", Computer Graphics Forum, Vol.27, No.2, pp.643-652, 2008.

[19] J. Novatnack and K. Nishino, "Scale-dependent 3D geometric features", In: Proc. of IEEE International Conference on Computer Vision, pp.1-8, 2007.

[20] A. Godil and A. I. Wagan, "Salient local 3D features for 3D shape retrieval", International Society for Optics and Photonics, Vol.7864, pp.78640S-78640S, 2011.

[21] B.W. Cheung and G. Hamarneh, "n-SIFT: ndimensional scale invariant feature transform", IEEE Transactions on Image Processing, Vol.18, No.9, pp.2012-2021, 2009.

[22] J. Sun, M. Ovsjanikov, and L. Guibas, "A concise and provably informative multi-scale signature based on heat diffusion", Computer Graphics Forum, Vol.28, No.5, pp.1383-1392, 2009.

[23] J.J. Koenderink and A.J. van Doorn, "Surface shape and curvature scales", Image and Vision Computing, Vol.10, No.8, pp.557-564, 1992.

[24] R. Song, Y. Liu, R. Martin, and P. Rosin, "3D point of interest detection via spectral irregularity diffusion", The Visual Computer, Vol.29, No.6-8, pp.695-705, 2013.

[25] C. Creusot, N. Pears, and J. Austin, "A machinelearning approach to keypoint detection and landmarking on 3D meshes", International Journal of Computer Vision, Vol.102, No.1-3, pp.146-179, 2013.

[26] L. Teran and P. Mordohai, "3D interest point detection via discriminative learning", In: Proc. of European Conference on Computer Vision, Springer, Cham, pp.159-173, 2014.

[27] T.H. Yu, O.J. Woodford, and R. Cipolla, "A performance evaluation of volumetric 3D interest point detectors", International Journal of Computer Vision, Vol.102, No.1-3, pp.180197, 2013.
[28] F. Tombari, S. Salti, and L. DiStefano, "Performance evaluation of 3D keypoint detectors", International Journal of Computer Vision, Vol.102, No.1-3, pp.198-220, 2013.

[29] S. Filipe and L.A. Alexandre, "A comparative evaluation of 3D keypoint detectors in an RGBD object dataset", In: Proc. of International Conference on Computer Vision Theory and Applications, pp.476-483, 2014.

[30] E. Boyer, A.M. Bronstein, M.M. Bronstein, B. Bustos, T. Darom, R. Horaud, I. Hotz, Y. Keller, J. Keustermans, A. Kovnatsky, R. Litman,J. Reininghaus, I. Sipiran, D. Smeets, P. Suetens, D. Vandermeulen, A. Zaharescu, and V. Zobel, "SHREC 2011: Robust feature detection and description benchmark", In: Proc. of Eurographics Conference on 3D Object Retrieval, pp.71-78, 2011.

[31] H. Dutagaci, C.P. Cheung, and A. Godil, "Evaluation of 3D interest point detection techniques via human-generated ground truth", The Visual Computer, Vol.28, No.9, pp.901-917, 2012.

[32] M. Everingham, L. Van Gool, C.K. Williams, J. Winn, and A. Zisserman, "The pascal visual object classes (VOC) challenge", International Journal of Computer Vision, Vol. 88, No.2, pp.303-338, 2010. 